\title{
„Leistung“ - Gestaltungsprinzip gesellschaftlicher und politischer Inklusion?
}

\author{
Oliver Gruber ${ }^{1, *}$ \\ 1 Institut für Politikwissenschaft, Universität Wien, Austria \\ * E-Mail: oliver.gruber@univie.ac.at
}

\section{Zusammenfassung}

Dem Leistungsparadigma als „Selbstdefinition moderner Gesellschaften“ liegt die Überzeugung zu Grunde, dass Rechte, Güter und Positionen auf Basis von Leistungen verteilt werden. In unterschiedlichen historischen Konstellationen als gesellschaftspolitisches Legitimationsprinzip vorgebracht, erlebt es derzeit eine Renaissance im Lichte von Wirtschaftskrise, steigender Arbeitslosigkeit, Bildungsreformdebatten und Integrationsherausforderungen. Ein klares Verständnis darüber, was als „Leistung“ zu verstehen ist, erweist sich jedoch seit jeher als schwierig und zunehmend schwieriger. Zudem birgt das Leistungsprinzip stets auch Exklusionsrisiken für darin adressierte Gruppen. Seine Ausweitung auf neue Politikfelder erlaubt nun, dieses Spannungsverhältnis neu zu betrachten und so Potentiale wie Grenzen gesellschaftlicher und politischer Inklusion durch Leistung zu analysieren. Dieses Editorial skizziert zunächst wesentliche ideengeschichtliche Etappen des Leistungsprinzips, zeigt zentrale Problem- und Fragestellungen des Themenheftes auf und stellt die darin versammelten Beiträge vor. .

\section{Schlüsselwörter}

Leistungsprinzip, Politische Theorie, Ideengeschichte, Inklusion/Exklusion

\section{„Meritocracy“ - A paradigm for sociopolitical inclusion?}

\begin{abstract}
Meritocracy is based on the belief that rights, goods and positions ought to be distributed on the basis of individual merit. As a sociopolitical claim it has been promoted in various historical constellations, currently it experiences a renaissance in light of economic crisis, increasing unemployment, recurrent debates on educational reforms and the challenges tied to immigrant integration. However, a clear-cut conception of what should be accepted as a "merit" has always been difficult and contested, and it tends to become ever more so. Moreover, meritocracy naturally goes along with exclusionary consequences for some groups addressed by its claim. Its expansion to new policy areas allows to re-examine these problems and thus to re-evaluate its inclusive potential. This editorial discusses the history of meritocratic thought and presents the special issue's research questions as well as the papers assembled in it.
\end{abstract}

\section{Keywords}

Meritocracy, political theory, history of ideas, inclusion/exclusion

\section{Danksagung:}

Die Entwicklung des Heftes erfolgte im Kontext des vom Jubiläumsfonds der Österreichischen Nationalbank geförderten Forschungsprojekts „Ein Staatssekretariat für Integration: Integrationspolitik in Bewegung?“ (Projektnummer: I5758). Besonderer Dank für seine Realisierung gilt der Projektleiterin Prof. Sieglinde Rosenberger sowie Peter Slominski für die umfangreiche redaktionelle Betreuung des Themenheftes und sämtlichen GutachterInnen für ihre Hinweise.

The author has declared that no competing interests exist. 
„The idea of meritocracy may have many virtues, but clarity is not one of them."

(Amartya Sen 1999: 5)

Dem Leistungsparadigma als „Selbstdefinition moderner Gesellschaften“ (Solga 2005, 23) liegt die Überzeugung zu Grunde, dass die Platzierung von Individuen in sozialen Hierarchien bzw. die Vergabe von Ressourcen ausschließlich auf Basis der dafür erbrachten Leistungen basieren darf. Seit jeher bewegt sich der Anspruch auf „Leistungsgerechtigkeit" im Spannungsfeld politischer Antagonismen, in unterschiedlichen historischen Konstellationen wurde "Leistung“ als zentrales politisches Legitimationsprinzip eingesetzt und aus gegensätzlichen politischen Lagern eingefordert.

Derzeit erlebt das Leistungsparadigma eine Renaissance im Lichte steigender Verteilungskämpfe. Wirtschafts- und Finanzkrise, wachsende Arbeitslosigkeit und Einkommensungleichheit, Reformdefizite im Bildungssystem ebenso wie Herausforderungen der Integration von MigrantInnen führen dazu, dass in politischen Debatten und Entscheidungen wieder offensiver auf Leistung als Legitimationskriterium zurückgegriffen wird (Bischoff 2005; Dahlgren 20I3; Degele/Winker 20II; Dröge et al. 2008; Gruber/Mattes 20I5; Hadjar 2008; Hagedorn 20I3; Hayes 2OI2; McNamee 2OI4; Neckel et al. 2008; Schatz 2006; Solga 2005; Voswinkel/Kocyba 2008). Das Leistungsparadigma scheint inzwischen auch für Politikfragen jenseits der traditionellen meritokratischen Triade „Bildung“, „Beruf“ \& „Einkommen“ (Kreckel 2004, 97) anschlussfähig geworden zu sein. Einerseits gerade weil es den Anspruch einer „Fundamentalnorm“ moderner Gesellschaften zu stellen versucht (Neckel et al. 2008, 4I), andererseits weil es eine scheinbar simple Lösung für die Steuerung gesellschaftlicher Diversität anbietet.

Bei näherer Betrachtung politischer Leistungsdiskurse zeigt sich hingegen, dass ein klares Verständnis darüber, was als „Leistung“ zu verstehen ist, immer schon schwierig war und zunehmend schwieriger wird (ibd., 42). Zudem birgt die Hervorhebung von Leistung als politischem Deutungs- und Gestaltungsrahmen stets auch das Potential exkludierender Folgen für die darin adressierten Gruppen. Die Ausweitung des Leistungsanspruchs auf neue Politikfelder erlaubt, dieses Spannungsverhältnis aus neuem Blickwinkel zu betrachten und damit Potentiale und Grenzen der gesellschaftlichen wie politischen Inklusion durch Leistung zu analysieren.

Diesem Anliegen geht das vorliegende Themenheft nach. Das folgende Editorial skizziert zunächst zentrale ideengeschichtliche Etappen des Leistungsprinzips. Darin werden klassische wie aktuelle Argumente für und wider meritokratische Gesellschaftsordnungen und -politik zusammengetragen und so zentrale Problem- und Fragestellungen des Themenheftes aufgezeigt. Im Anschluss daran werden der Aufbau des Heftes und die darin versammelten Beiträge dargestellt.

\section{Ideengeschichtliche Linien eines gesellschaftspolitischen Leistungsprinzips}

\subsection{Vormoderne Wurzeln meritokratischen Denkens}

Unter dem heute für das gesellschaftspolitische Leistungsparadigma etablierten Terminus der „Meritokratie“ (lat. meritum, Verdienst; griech.: kratein, Herrschaft) beschrieb erstmals Michael Young (I958/I996) eine dystopische Zukunftsgesellschaft, in der Individuen ausschließlich auf Basis ihrer Leistung (von ihm definiert als IQ + Anstrengung) zu sozialen Rängen wie Bildungspositionen oder Arbeitsplätzen zugewiesen werden. Obwohl satirisch verwendet, hielt der Begriff Einzug in die politisch-soziologische Sprache als Beschreibung eines Tauschprinzips individueller Leistung gegen entsprechende Be- oder Entlohnung (Celarent 2009). Die Wurzeln eines auf Leistungsprinzipien basierenden Denkens reichen freilich wesentlich weiter in die gesellschaftspolitische Ideengeschichte zurück.

Bereits Platon skizziert in der Politeia einen meritokratischen Aufbau gesellschaftlicher Ordnung, in welchem die Platzierung des Individuums entsprechend seiner Geschicke und Leistungsmöglichkeiten erfolgen solle (Platon, Politeia, 4. Buch, 433a). Folgerichtig kommen auch bei der Auswahl der Staatsführung in seinen Augen nur die besten und klügsten Personen in Frage - Platons Argument für die Philosophenherrschaft als Mittel zur Verwirklichung des guten Staates (ibd., 5. Buch, 473-476). Auch Aristoteles beschreibt im Wesentlichen eine meritokratische Vorstellung gesellschaftlicher und politischer Ordnung, in der die berufliche und soziale Stellung seiner Bürger von deren „natürlichen Qualitäten“ abhängen soll (Artistoteles, Politik 7. Buch, Kap. 9). Anders als in der neuzeitlichen Verwendung des Leistungsprinzips zieht er es - ähnlich wie Platon - als Argument für die klare Segmentierung der Gesellschaft auf Basis individueller Leistungen heran (ibd. Kap. Io) - was auch ihn zur Befürwortung einer Herrschaft der „Besten“ (aristoi) im Dienste der Verwirklichung des guten Lebens aller führt (ibd., 3.Buch, Kap. 5-I8).

Außerhalb des europäischen Raumes gilt insbesondere die politische Kultur Chinas als eine frühe Keimzelle meritokratischen Denkens: Hier entstand der Anspruch einer „Hochschätzung der Tüchtigen“ im Zuge der Auflösung der askriptiven, auf verwandtschaftlicher Abstammung begründeten aristokratischen Ordnung seit dem 7. Jahrhundert vor Christus (Bell 2OI2, 9). Leistungsprinzipien gingen letztlich 
auch in das religiöse Selbstverständnis über, der Konfuzianismus etablierte schon früh den Gedanken eines Vorrangs der Leistung anstelle der Geburtsrechte. Bereits im 2. Jahrhundert vor Christus existierten im Chinesischen Kaiserreich Testsysteme, auf deren Basis allen Befähigten der Zugang zum Staatsdienst ermöglicht wurde (Franke 1972, I-2).

Dass die gesellschaftspolitische Praxis in Europa zunächst nicht vom Leistungsanspruch durchflutet wurde, führen Voswinkel/Kocyba $(2008,22)$ auf seine gesellschaftliche Verfasstheit zurück. Europas vormoderne Gesellschaften mit ihrer stratifizierten Ordnung konnten noch kein gesellschaftsübergreifendes Leistungsprinzip etablieren. Leistung war zwar ein Element der ökonomischen Alltagsmoral der Menschen, jedoch waren die Realitäten der gesellschaftlichen Teilbereiche von der Spitze bis zur Basis derart verschieden, dass sich noch keine allgemeine Leistungswährung herauszubilden vermochte. Diese Entwicklung nahm in Europa erst im Wechsel zur Moderne und dem Übergang zu funktional differenzierten Gesellschaftsstrukturen konkretere Gestalt an (Luhmann 1998, 678ff.).

\subsection{Aufschwung des Leistungsparadigmas im Über- gang zur Moderne}

Die Erschütterungen der tradierten feudalen und aristokratischen Ordnung im Europa und Amerika seit dem I7. Jahrhundert katalysierten auch hier meritokratische Argumente unter politischen TheoretikerInnen. Aufklärungsphilosophen wie Voltaire formulierten die Überzeugung, dass erst durch das Leistungsprinzip die Ordnung des Ancien Régime überwunden werden könne (Voltaire selbst war ein großer Bewunderer des zentralisierten Staatssystems des Imperialen China und seiner meritokratischen Elemente gewesen) (Switalsky 20I4, 45). Auch die Amerikanischen Gründerväter waren getragen von dem Gedanken, eine Balance zwischen Volkssouveränität und zivilgesellschaftlicher Partizipation einerseits sowie meritokratisch gestalteten politischen Institutionen andererseits zu realisieren, um so die "natural aristocracy of talent and virtue“ (Thomas Jefferson) zu fördern (Bergruen/Gardels 20I2, 25f.). Liberale Elitentheoretiker des 19. Jahrhunderts wie Mill oder Tocqueville versuchten, das Ideal der Demokratie mit jenem des Leistungsprinzips zu verbinden (Bell 20I2, IO) - prototypisch in Mills Vorschlägen zur meritokratisch besetzten legislativen Kammer der Staatsmänner oder zum gestaffeltem Pluralstimmrecht mit Stimmbegünstigungen für befähigte BürgerInnen (Mill I862, Kap.8).

Zugleich befruchteten die Ideale der Aufklärung und des Liberalismus ein sich emanzipierendes Bürgertum in seinem Kampf gegen die aristokratischen Privilegi- en. Die bürgerlichen Revolutionen ab dem I7. Jahrhundert standen unter dem Banner des Leistungsprinzips: der gesellschaftliche Status und Wohlstand sollte nicht länger ein leistungsloser Erbanspruch des Adels und von der Abstammung sondern von der - materiell verstandenen - Leistungsfähigkeit individueller Personen abhängig sein (Henning I98I; Walter/Marg 2013, 97ff.). Damit in Zusammenhang standen nicht zuletzt auch religiöse Veränderungen, der sich ausbreitende Protestantismus schrieb dem Leistungsprinzip eine zentrale Bedeutung zu. Die dort angelegte Leistungsethik fasste Arbeit zwar weniger unter dem Gesichtspunkt fairer Entlohnung oder als Kriterium gesellschaftlicher Hierarchisierung auf, sondern vielmehr als göttlich vorgesehene Pflicht des Menschen. Die Verbindung des protestantischen Leistungsgedankens mit dem sich herausbildenden bürgerlichen Standesbewusstsein auf Basis des durch Leistung erworbenen Wohlstands machte es aber für die Entwicklung meritokratischer Argumentation zu einem wesentlichen Impuls (Kuhlemann 2000, 305f.) - Max Weber (1904/2006) sollte ihm später gar eine Schlüsselrolle für den Aufstieg des "Geistes des Kapitalismus“ zuschreiben, eine viel kritisierte und bis heute kontroversielle These (vgl. Guttandin 1998).

Unter umgekehrten Vorzeichen doch mit ähnlichen Argumenten griff im 19. Jahrhundert schließlich auch die Arbeiterbewegung das Leistungsprinzip für sich auf. Das infolge der industriellen Revolution angewachsene Industrieproletariat richtete nun den Vorwurf der fehlenden Leistungsgerechtigkeit an das Besitzbürgertum (Schatz 2004). Mit dem Anspruch gerechter Entlohnung für die (körperliche) Arbeitsleistung der Arbeiterschaft ging der Vorwurf einher, man selbst erbringe die wertschöpfenden Leistungen, während die Kapitaleigentümer den überwiegenden Anteil des dadurch erzielten Gewinns einstreichen (Bischoff 2005). Die bürgerliche Reaktion darauf verwendete das Leistungsprinzip nun in umgekehrter Form, und zwar als Argument für bestehende Hierarchien. Ihm zufolge läge höherem Status und Wohlstand eben auch bedeutsamere Leistung zugrunde, Kritik daran bringe lediglich Sozialneid zum Ausdruck. Darin offenbarte sich die inhärente Ambivalenz des Leistungsprinzips: es diente nun zugleich als Instrument der Kritik wie der Rechtfertigung bestehender Verhältnisse (Voswinkel/Kocyba 2008, 22).

Das Leistungsprinzip festigte im Zuge dieser Etappen also seinen Stellenwert als Legitimationsgrundlage für soziale Ungleichheiten. Gestritten wurde zwar über die gerechte Definition von Leistung, jedoch nicht mehr um dessen grundsätzliche Sinnhaftigkeit als Prinzip für die Verteilung von Ressourcen und gesellschaftlichem Status. 
1.3 Multiple Kritiken am Leistungsprinzip im fordistischen Zeitalter

Im 20. Jahrhundert gewannen schließlich systematischere Kritiklinien an meritokratischen Legitimationsstrukturen an Gewicht. Vor allem Michael Youngs satirische Abrechnung mit der Meritokratie traf den Nerv des Zeitgeists am Höhepunkt der fordistischen Ära, zunächst im englischsprachigen, mit einiger Verzögerung aber auch im deutschsprachigen Raum (Walter/ Marg 2013, I06). Die „Leistungsgesellschaft" wurde zum Streitobjekt akademischer wie politischer Debatten, die Kritik daran kam interessanterweise aus unterschiedlichen politischen Lagern und Denkschulen:

\section{"Sozialistische Torpedierung der Marktgesetze“}

Friedrich Hayek stellte aus neoliberaler Perspektive das Leistungsprinzip fundamental in Frage und meinte, auf seine normative Legitimationskraft verzichten zu können (Schatz 2006, 3002). Konkret kritisierte er, dass durch ein aufwandsorientiertes Leistungsprinzip der Verdienst einer Person fälschlicherweise mit dem Marktwert der von ihr produzierten Leistungen vertauscht werde. Ein kaum messbares und schwer praktikables Organisationskriterium werde damit zum normativen Gesellschaftsprinzip erhoben. Im Gegensatz dazu hielt Hayek in der Tradition der Markttheoretiker à la Adam Smith einzig eine marktwirtschaftliche Ordnung aus Angebot und Nachfrage für umsetzbar. Darin sei der Verdienst einer Person allein durch ihren Marktwert zu bemessen, also dem was sie anzubieten habe und wofür andere bereit sind etwas zu bezahlen (Hayek 1960/2005, Kap.6). Meritokratie, so Hayek später, sei nur ein „trojanisches Pferd“ des Sozialismus im Kampf um soziale Gerechtigkeit (Hayek 1979/2004, 287).

\section{Disziplinierungsinstrument der Leistungsoligarchie}

Von Seiten der politischen Linken wurde das Leistungsprinzip wiederum für seinen herrschaftserhaltenden Charakter kritisiert. Marx (I89I/I984) selbst hatte schon betont, dass die reine Belohnung der Leistung die ungleichen Chancen von Individuen naturalisiere, anstatt sie zu überwinden. Daran knüpften im 20. Jahrhundert unter anderem Kritische TheoretikerInnen an. Herbert Marcuse (1965) etwa verband marxistische mit Freud'schen Überlegungen zu dem Argument, das Leistungsprinzip sei die derzeit bestehende Ausprägung des Freud'schen Realitätsprinzips und werde von den meisten Menschen nur noch als „entfremdete Arbeit" infolge der (kapitalistischen) Unterdrückung von Mensch und Natur erlebt. Erst eine Abkehr davon könne, so Marcuse, bestehende Herrschaftsverhältnisse überwinden und zugleich eine Kultur der Freiheit für das menschliche Lustprinzip etablieren. Claus Offe (1970, I65) vertiefte die marxistische Kritik dahingehend, dass nicht „die unvollkommene Verwirklichung, sondern gerade (...) die Konsequenzen seiner allzu perfekten Durchsetzung“" das Leistungsprinzip kritikwürdig machen. Die Illusion einer gerechten, durchgehenden Leistungsskala für Berufsarbeit sei illusorisch. Das Leistungsprinzip sei kein rationales „Verfahren zur Allokation unterschiedlicher individueller Arbeitskraft" mehr, sondern „eine Disziplinierungstechnik, welche die Loyalität mit herrschenden Interessen und Lebensformen prämiert, kulturelle Spaltungen perpetuiert und den Schein einer objektiven oder, technischen' Begründbarkeit organisatorischer Hierarchien hervorbringt und stabilisiert." (ibd., I66) Statt zur Überwindung ständischer Strukturen kommt es also zur Ersetzung der einstigen Hierarchien mit jenen einer Leistungsoligarchie (Hayes 2012, 282).

\section{Intersektionales Diskriminierungssystem}

Schließlich bildeten auch ProponentInnen von Affirmative Action Policies für Frauen und ethnische Minderheiten eine weitere Quelle der Kritik am Leistungsprinzip. Sie erneuerten die Kritik am hegemonialen Charakter des Leistungsprinzips, dieses diene nur zur Stützung der dominierenden gesellschaftlichen Gruppe und stehe Formen positiver Diskriminierung im Wege (Miller 1996, 279). Damit drückten sie zwar keine Fundamentalablehnung am Leistungsprinzip aus, öffneten allerdings den Blick für dessen diskriminierende Effekte auch jenseits der historischen Konflikte um die Kategorie Klasse. Den intersektionalen Barrieren des Leistungsprinzips könne erst mit dem Abbau struktureller Diskriminierungen begegnet werden, ein ernstgenommenes Leistungsprinzip habe daher bereits an der Inputseite der Leistungskette anzusetzen (Degele/Winker 20II; Ringer 1976; I. M. Young 1990).

\section{Makrogerechtigkeit statt Individualisierung}

Eine weitere Kritiklinie am Leistungsprinzip formulierte Daniels (1978), der auf die Konsequenzen zwischen Mikro- vs. Makroproduktivitätserwägungen hinwies. Während bei der Vergabe von Berufspositionen mit Leistungen auf der individuellen Mikroebene (die besten Stellen für die besten Personen) argumentiert werde, ignoriere dies den gesamtgesellschaftlichen Bedarf an der Besetzung aller Jobs bzw. der Platzierung aller Individuen. Die „Individualisierung“ des Leistungsprinzips lasse das soziale Ganze außer Acht, ja es sei aus Makroproduktivitätssicht sogar unproduktiv. Denn es werde nicht der maximale Nutzen der verfügbaren Leistungsressourcen aller Personen genutzt, sondern nur die optimale Ausschöpfung der Leistungsfähigsten bei gleichzeitigem Verlust der Leistungspotentiale anderer erreicht. Aus ähnlichen Erwägungen formulierte auch John Rawls seine liberale „Theorie der Gerechtigkeit“ in Abgrenzung zu meritokratischen Gesellschaftsvorstellungen. Während dort Chancengleichheit nur darin 
bestehe, „die weniger Glücklichen in dem persönlichen Wettkampf um Einfluß und gesellschaftliche Stellung hinter sich zu lassen“ (Rawls 1979/2OI4, I28), plädierte er für eine egalitäre Gesellschaftsordnung. In dieser seien die Ungleichheiten individueller Startbedingungen zur Förderung der Lebensbedingungen aller zu nutzen, sodass "niemand von seinem zufälligen Platz in der Verteilung der natürlichen Gaben oder seiner Ausgangspositionen in der Gesellschaft Vor- oder Nachteile hat, ohne einen Ausgleich zu geben oder zu empfangen." (ibd., I23)

\subsection{Das Leistungsparadigma heute: Zwischen Konsoli- dierung und Erosion}

In der postfordistischen Ära mehren sich neben den theoretischen inzwischen auch die empirischen Krisendiagnosen gegenüber dem Leistungsprinzip. Langzeitarbeitslosigkeit, prekäre Beschäftigungs- und Einkommensstrukturen und der Rückbau des Wohlfahrtsstaates - um nur einige Entwicklungen zu nennen - lassen die Kluft zwischen Ideal und Wirklichkeit auch in westlichen Demokratien deutlicher werden und die empirische Gültigkeit des Leistungsprinzips zunehmend fraglich erscheinen (Schatz 2006, 3003). Hinzu kommen Befunde "meritokratischer Paradoxa", wenn selbst die Etablierung leistungsbasierter Regeln in Organisationen oftmals nicht zur Beseitigung diskriminierender Behandlung führt, sondern sie indirekt sogar unterstützen kann (Castilla 2008; Castilla/Benard 20IO; Elvira/ Graham 2002).

Trotz allem scheint die normative Wirkmächtigkeit des Prinzips nach wie vor ungebrochen. Dröge et al. $(2008,8)$ diagnostizieren gar, dass es mehr denn je in die „öffentliche wie wissenschaftliche Debatte zurückgekehrt (ist) - allerdings unter gewandelten Vorzeichen". Während die Debatte der I970er-Jahre noch ein Ende der Leistungsgesellschaft vorausgesagt hatte, werde sie heute nicht mehr grundsätzlich in Frage gestellt, sondern nur noch hinsichtlich partieller Dysfunktionalitäten in einzelnen Teilbereichen kritisiert bzw. in adaptierten Konzepten von Meritokratie fortgeführt. Bell etwa argumentiert seit den I970er-Jahren für eine "gerechte Meritokratie", in der zwar alle Personen gleichberechtigtes Anrecht auf Grundversorgung und Grundeinkommen hätten, dadurch aber keineswegs einen Gleichheitsanspruch auf allen Gebieten erlangen sollten, schon gar nicht hinsichtlich ihrer sozialen Platzierung oder Einkommen (diese seien weiterhin rein leistungsbasiert zu vergeben) (Bell I972, 65-67; 2012). Miller (1996, 279) hält das Prinzip der Meritokratie sogar in seiner Reinform für nach wie vor vereinbar mit modernen marktwirtschaftlichen Gesellschaften. Die Hindernisse lägen eher in unseren Traditionen, in Geschlechtervorurteilen, in Rassismen und in den beziehungs- und netzwerkfördernden Mustern bei der Vergabe von Ressourcen - sie alle hält er durch Bildung bzw. gesetzliche und institutionelle Vorkehrungen für überwindbar, ohne aber dafür das Leistungsprinzip in Frage stellen zu müssen. Und McNamee und Miller (McNamee 20I4; McNamee/Miller 2004) diagnostizieren zwar, dass das Leistungsprinzip heute nur noch ein "meritokratischer Mythos" sei, plädieren aber ähnlich für dessen (Wieder-)Herstellung, unter anderem durch Maßnahmen der Anti-Diskriminierung, der Affirmative Action, der philantropischen Wohlfahrt oder der egalitäreren Besteuerung.

Inwieweit mit derartigen Vorschlägen die Grundproblematiken einer meritokratisch organisierten Gesellschaft effektiv eingehegt werden können, bleibt angesichts der Persistenz aktueller Krisensymptome zwar weiterhin fraglich. Als normativer Rahmen scheint das Leistungsprinzip jedoch den Rang einer Fundamentalnorm eingenommen zu haben, dem heute kaum ein soziales Subsystem mehr vollständig entgeht (Neckel et al. 2008, 4I). Seine Wettbewerbssemantik kommt in elitären „Exzellenzdiskursen“ ebenso zum Ausdruck wie in popkulturellen Unterhaltungsformaten nach dem Prinzip „The winner takes it all“. Als Legitimationsprinzip ist der Leistungsgedanke also tief eingeschrieben in unser Verständnis von Gerechtigkeit (siehe dazu den Beitrag von Kai Dröge in diesem Heft), was es auch so vielseitig adressierbar macht, sowohl für top-down Legitimation wie auch für bottom-up Kritik (McNamee/Miller 2004; Scully 1997).

\subsection{Synthese: Problemstellungen des Leistungsprinzips}

Die kursorische ideengeschichtliche Rekonstruktion des Leistungsprinzips verdeutlicht dessen konfliktreiche und nach wie vor verhandelte Natur. Fasst man grundlegende Spannungspunkte zusammen, so stechen einige Problemstellungen hervor, auf die Meritokratieanalysen bis heute besonders Augenmerk legen. Sie leiten folglich auch die Analysen des vorliegenden Themenheftes an:

- Individualisierung durch Leistung: Meritokratische Gesellschaftskonzepte sind durch einen individualistischen Zugang charakterisiert. Individuelle Leistungsfähigkeiten bestimmen den Wert und Status einer Person in diesem Gefüge. Dies weist dem Einzelnen zwar ein hohes Maß an eigener Gestaltungsmacht zu, macht ihn allerdings auch zum alleinigen Verantwortlichen im Falle des Misserfolgs und damit zum Trägervon Exklusionskosten - mit all den soziopsychologischen Folgen, denen sich eine Analyse von Leistungsordnungen zu widmen hat (Voswinkel/Kocyba 2008).

- Leistung als kontingentes Prinzip: Obgleich das Leistungsprinzip seine Attraktivität gerade aus der abstrakten Natur als gesellschaftliches Ordnungsprinzip gewinnt, beruht seine praktische Anwendung stets 
auf spezifischen, durch den Anwendungskontext definierten Leistungskriterien. Deren Festlegung folgt in der Regel keinen demokratischen Verfahren oder egalitären Zielen, sondern ist ein Ausdruck von Machtverhältnissen, die bestimmte Gruppen begünstigen und andere benachteiligen (Miller 1996) - ihre Offenlegung ist eine wesentliche Aufgabe von Meritokratieforschung.

- Messbarkeitsgrenzen von Leistung: Praktische Schwierigkeiten in der Anwendung von Leistungsordnungen entstehen dadurch, dass etablierte Maßstäbe kaum die Vielzahl an persönlichen Bedingungen berücksichtigen können, unter denen Personen Leistung zu erbringen haben. Herangezogen werden folglich nur bestimmte, objektivierbare Kriterien, die auf individuelle Lebenssituationen angelegt werden. Dies verstärkt nicht nur Gerechtigkeits- sondern auch Ungerechtigkeitsempfinden der Betroffenen und stellt somit ein zentrales Beurteilungskriterium für die Legitimität meritokratischer Ordnungen dar (Young I990).

- Naturalisierung sozial produzierter Leistungsungleichheit: Werden meritokratische Argumente zur Rechtfertigung bestehender Statushierarchien verwendet, so drohen dadurch Ungleichheitsstrukturen als legitime Rangordnung der Leistungsfähigkeiten von Menschen naturalisiert zu werden. Soziale Hierarchie reflektiert in dieser Logik dann die Qualitäten von Individuen, weniger erfolgreiche Personen - in einem Leistungswettbewerb also die Mehrheit - erscheinen zu Recht an ihrem Platz (Hayes 20I2). Die Offenlegung derartiger Konstruktionen ist eine wesentliche Aufgabe kritischer Studien des Leistungsprinzips.

- Leistung zwischen Aufwands- und Erfolgsprinzip: Eine sich in Leistungsanalysen zunehmend zeigende Problematik betrifft die Kluft zwischen Aufwands- und Ergebnisentschädigung. Insbesondere im Kontext erodierender Normalarbeitsverhältnisse aber auch im bildungspolitischen Zusammenhang ist zu beobachten, dass nicht mehr die investierte Anstrengung als Kriterium für die Beurteilung von Leistung gilt, sondern der Erfolg des Endprodukts zum zentralen Maßstab erhoben wird (Sen 1999). Damit werden einerseits Produktions- und Sozialkosten auf Individuen abgewälzt, andererseits die Begünstigung ressourcenstarker Personen gegenüber jenen mit schlechteren Voraussetzungen verfestigt (Hagedorn 2OI3).

- Begrenzungsnotwendigkeit des Leistungsallanspruchs: Welche Güter und Positionen lassen sich überhaupt sinnvoll auf Basis meritokratischer Distributionslogik verteilen? Diese Frage prägt maßgeblich die Meritokratieanalysen jenseits der traditionellen Forschungsbereiche Bildung und Arbeit. Denn wo die Anwendung eines reinen Leistungsprinzips über die Vergabe von beruflichen Positionen, Löhnen und Bildungsabschlüssen hinausreicht, können problematische Folgen entstehen. Werden etwa sensible Bereiche wie Gesundheitsversorgung oder Alterssicherung meritokratischen Ordnungen unterworfen, so bleiben fundamentale (Über-)Lebenschancen von der Marktgängigkeit individueller Fähigkeiten abhängig - und damit in hohem Maße exklusiv (Miller I996).

- Leistung zwischen individueller und kollektiver Gerechtigkeit: Wird politische Verantwortung als Gemeinwohlverpflichtung verstanden, so bleibt nicht zuletzt in Frage zu stellen, inwieweit die gesamtgesellschaftlichen Kosten einer Meritokratie tatsächlich ihren Nutzen übertreffen. Gemessen an ihrem eigenen Anspruch bleibt dies eine Voraussetzung für die Legitimität von Leistungsprinzipien (Daniels I978).

\section{Aufbau des Themenheftes}

\subsection{Erkenntnisinteresse}

Vor dem Hintergrund dieser Problemstellungen geht es dem vorliegenden Themenheft um eine empirisch fundierte Auseinandersetzung mit dem Leistungsprinzip in drei verschiedenen Politikfeldern. Dabei werden einerseits Erfahrungen aus traditionell durch Leistungssemantiken und -ordnungen geprägten Feldern wie der Arbeitsmarkt- und Bildungspolitik untersucht, andererseits auch das Politikfeld der Integration von MigrantInnen berücksichtigt, in dem derartige Prinzipien erst in jüngerer Vergangenheit Einzug gehalten haben. Ein solcher Vergleich stellt nach unserem Kenntnisstand bislang ein Forschungsdesiderat dar. Auf Basis einer länder- und politikfeldübergreifenden Zusammenstellung von Beiträgen vereint das Themenheft daher AutorInnen aus Österreich, Deutschland und Luxemburg, die ihre bisherige Forschung aus Bildungs-, Arbeitsmarktund Integrationspolitik unter dem Gesichtspunkt der Inklusions-/Exklusionsdynamiken des Leistungsansatzes vertiefen. Damit leistet das Themenheft einen sowohl theoretischen wie empirischen Beitrag zur Bearbeitung einer virulenten Herausforderung in Politikwissenschaft und Soziologie.

Mit der Analyse der in diesen Politikfeldern angewendeten Leistungskriterien, der darüber geführten politischen Konflikte sowie der Erfahrungen davon betroffener Personengruppen versucht das Heft, Potentiale und Grenzen eines Leistungsprinzips als gesellschaftspolitischen Inklusionsmechanismus zu skizzieren. Im Zentrum steht die Frage, ob meritokratische Prinzipien in gleichem Maße für unterschiedliche Politikfelder als Ordnungsprinzip geeignet sind bzw. wo Grenzen für de- 
ren Anwendbarkeit bestehen. Welche Verständnisse von Leistung und Gerechtigkeit liegen diesen Politikfeldern jeweils zu Grunde? Welche inkludierenden, welche exkludierenden Momente sind mit der Anwendung eines Leistungsprinzips jeweils verbunden? Und inwieweit unterscheiden sich diskursive Leistungssemantiken von substantiellen leistungspolitischen Maßnahmen?

\subsection{Zu den Beiträgen}

Der Beantwortung dieser Fragen widmet sich das Themenheft im Rahmen von vier Beiträgen:

Den Anfang macht eine konzeptionelle Analyse des Leistungsparadigmas aus sozialintegrativer Perspektive. Kai Dröge beschäftigt sich in seinem Text ausgehen von Axel Honneths Anerkennungstheorie mit dem normativen Anspruch und den Grenzen des Leistungsprinzips. Er skizziert dessen formalen Kern an Kriterien und geht auf zwei daraus resultierende Konsequenzen für dessen Integrationskraft ein: Zum Einen auf die Funktion des mit dem Leistungsprinzip verbundenen Deutungsüberschusses als normativer Ressource unterschiedlicher sozialer Gruppen in ihrem Kampf um Anerkennung und damit auch gesellschaftliche Integration. Zum Anderen auf die ideologische und widerspruchsfördernde Natur des Leistungsprinzips, dessen Durchsetzung stets auch durch Politiken der Missachtung und Ausschließung gekennzeichnet ist. Anhand der Befunde aus Gruppendiskussionen mit Berufstätigen unterschiedlicher sozialer Milieus und Tätigkeitsbereiche werden diese konzeptionellen Überlegungen empirisch untermauert.

Auch der zweite Beitrag des Themenheftes widmet sich der Arbeitswelt, allerdings unter dem besonderen Gesichtspunkt der Umstrukturierung des öffentlichen Dienstleistungswesens. Am Beispiel der Österreichischen Post zeigt Jörg Flecker, wie durch Privatisierung und Kommerzialisierung die Zweckbestimmung von Organisationen neu festgelegt und ein geändertes Verständnis dessen durchgesetzt wird, was als Leistung gilt und was nicht. Die dadurch nur noch partielle Inklusion und die teilweise Prekarisierung von Arbeit werden durch die vielfachen Spaltungen der ArbeitnehmerInnenschaft gestützt, während sie diese gleichzeitig verstärken. Auf Basis von Interviews mit betroffenen MitarbeiterInnen verdeutlicht der Beitrag einen tiefgreifenden Wandel der Anerkennungsverhältnisse, wenn in der Legitimation von Ungleichheit Erfolg an die Stelle von Leistung tritt, während Normalarbeitsleistungen soziale Wertschätzung vorenthalten bleibt. Abhängig von der beruflichen Identität der MitarbeiterInnen wird dies in unterschiedlichem Ausmaß als Verletzung von Fairness und Gerechtigkeit wahrgenommen.

Mit dem dritten Beitrag wechselt das Heft sein Beobachtungsfeld und setzt sich mit dem Verhältnis von Chancengleichheitsvorstellungen und Leistungsmoti- ven in der Bildungspolitik auseinander. Andreas Hadjar und Barbara Rothmüller gehen in ihrem Beitrag zunächst theoretisch der Frage nach, inwieweit das Konzept der Gesamtschule bzw. gesamtschulartiger Bildungssysteme - in Abgrenzung von streng gegliederten bzw. stratifizierten Systemen - den normativen Ansprüchen an Chancengleichheit zu entsprechen vermögen. Aufbauend darauf rekonstruieren sie in einer historischen Analyse der politischen Debatten um die gescheiterte Einführung einer Luxemburgischen Gesamtschule die ungleiche und von ideologischen Lagern konträr aufgeladene Verwendung des Gleichheitsbegriffs sowie die daraus resultierenden gegensätzlichen Verständnisse von Leistungsorientierung. Im Rahmen dessen verdeutlichen sie, wie in diesen Konfliktpositionen nicht nur konträre Vorstellungen über die Leistungsförderung von SchülerInnen zum Ausdruck kommen, sondern auch fundamentale Statuskonflikte der LehrerInnenschaft über ihre eigenen Berufshierarchien ausgetragen werden.

Der letzte Beitrag des Themenheftes verlässt schließlich die traditionell durch Leistungsdebatten geprägten Politikfelder und wendet sich der Einführung des Leistungsprinzips im noch jungen Politikfeld der Integration von MigrantInnen zu. Am Beispiel der Österreichischen Integrationspolitik seit Etablierung eines Staatssekretariats für Integration analysieren Oliver Gruber, Astrid Mattes und Jeremias Stadlmair die Kontinuitäten und Brüche zwischen der diskursiven Verwendung von Leistung als politischem Narrativ und seiner Umsetzung in substantielle Integrationspolicies. Sie zeigen, wie sich der Ansatz auf traditionelle Leistungskriterien der Arbeitsmarkt- und Bildungspolitik stützt und inwieweit er damit eine durch askriptive Merkmale (wie Ethnizität, Nationalität, Sprache) kategorisierte Personengruppe effektiv zu erfassen vermag. Dies erlaubt schließlich eine kritische Einschätzung der immanenten Ambivalenzen eines Leistungskonzeptes in der Integrationspolitik einerseits sowie eine Gegenüberstellung der damit einhergehenden Inklusions- und Exklusionspotentiale andererseits.

In ihrer Zusammenstellung verdeutlichen die Beiträge somit Spezifika wie Gemeinsamkeiten der Politikfelder in der Anwendung von Leistungsprinzipien und leisten damit einen Beitrag zur Bündelung einer bislang weitgehend auf einzelne Politikfelder begrenzten Debatte. Dies kann freilich nur ein Anstoß sein, dem weitere integrative Auseinandersetzungen hoffentlich folgen werden. 


\section{Literatur}

Bell, Daniel A. (1972). On Meritocracy and Equality, in: Public Interest, Vol. 29, 29-68.

Bell, Daniel A. (2012). Meritocracy Is a Good Thing, in: New Perspectives Quarterly, Vol. 29(4), 9-I8.

Bergruen, Nicholas/Nathaniel Gardels (2012). Political Meritocracy and Direct Democracy: A Hybrid Experiment, in: New Perspectives Quarterly, Vol. 29(4), 24-4I.

Bischoff, Joachim (2005). Das Ende des Neoliberalismus und die Zukunft der Wirtschaftsdemokratie, in: UTOPIE kreativ, Vol. 173, 200-2II.

Castilla, Emilio J. (2008). Gender, race, and meritocracy in organizational careers, in: American Journal of Sociology, Vol. II3(6), I479-I526.

Castilla, Emilio J./Stephen Benard (2010). The Paradox of Meritocracy in Organizations, in: Administrative Science Quarterly, Vol. 55(4), 543-576.

Celarent, Barbara (2009). The Rise of the Meritocracy, I870-2033 by Michael Young, in: American Journal of Sociology, Vol. II5(I), 322-326.

Dahlgren, Robert L. (2013). Meritocracy and its Discontents, in: Theory \& Research in Social Education, Vol. $4 \mathrm{I}(2), 280-284$.

Daniels, Norman (1978). Merit and Meritocracy, in: Philosophy \& Public Affairs, Vol. 7(3), 206-223.

Degele, Nina/Gabriele Winker (20II). ,Leistung muss sich wieder lohnen'. Zur intersektionalen Analyse kultureller Symbole, in: Katharina Knüttel/Martin Seeliger (Hg.): Intersektionalität und Kulturindustrie. Zum Verhältnis sozialer Kategorien und kultureller Repräsentationen, Bielefeld, 25-53.

Dröge, Kai/Kira Marrs/Wolfgang Menz (2008). Rückkehr der Leistungsfrage. Leistung in Arbeit, Unternehmen und Gesellschaft - zur Einleitung, in: Kai Dröge/Kira Marrs/Wolfgang Menz (Hg.): Rückkehr der Leistungsfrage. Leistung in Arbeit, Unternehmen und Gesellschaft, Berlin, 7-I8.

Elvira, Marta M./Mary E. Graham (2002). Not just a formality: Pay system formalization and sex-related earnings effects, in: Organization Science, Vol. I3(6), 6oI-6I7.

Franke, Wolfgang (1972). The reform and abolition of the traditional Chinese examination system Cambridge, MA.

Gruber, Oliver/Astrid Mattes (2015). Das Narrativ ,Integration durch Leistung. Neuansatz staatlicher Integrationspolitik?, in: Karin B. Schnebel (Hg.): Europäische Minderheiten. Im Dilemma zwischen Selbstbestimmung und Integration, Wiesbaden, 89-II6.

Guttandin, Friedhelm (1998). Einführung in die „Protestantische Ethik" Max Webers, Wiesbaden.

Hadjar, Andreas (2008). Meritokratie als Legitimationsprinzip. Die Entwicklung der Akzeptanz sozialer
Ungleichheit im Zuge der Bildungsexpansion, Wiesbaden.

Hagedorn, Udo (20I3). Leistung oder Erfolg? Berufsbildungspolitische Deutungsmuster und die prinzipielle Ausrichtung der Berufsausbildung, in: Karin Büchter/Sandra Bohlinger/Tade Tramm (Hg.): Berufs- und Wirtschaftspädagogik - online, Nr. 28: Ordnung und Steuerung der beruflichen Bildung. Internet: http:// www.bwpat.de/ausgabe25/hagedorn_bwpat25.pdf (Zugriff: I4.03.2016).

Hayek, Friedrich August (1960/2005). Die Verfassung der Freiheit, Tübingen.

Hayek, Friedrich August (1979/2004). Recht, Gesetz und Freiheit, Tübingen.

Hayes, Christopher L. (2012). Twilight of the Elites: America After Meritocracy, New York, NY.

Henning, Hansjoachim (198I). Sozialgeschichte, in: Willi Albers (Hg.): Handwörterbuch der Wirtschaftswissenschaften, Stuttgart, Tübingen, Göttingen, 66I-688.

Kreckel, Reinhard (2004). Politische Soziologie der Ungleichheit ( 3 ed.), Frankfurt, New York.

Kuhlemann, Frank-Michael (2000). Bürgertum und Religion, in: Peter Lundgreen (Hg.): Sozial- und Kulturgeschichte des Bürgertums: eine Bilanz des Bielefelder Sonderforschungsbereichs (1986-1997), 293-318.

Luhmann, Niklas (1998). Die Gesellschaft der Gesellschaft, Frankfurt.

Marcuse, Herbert (1965). Triebstruktur und Gesellschaft. Ein philosophischer Beitrag zu Sigmund Freud, Frankfurt.

Marx, Karl (189i/1984). Kritik des Gothaer Programms. Randglossen zum Programm der deutschen Arbeiterpartei, Berlin.

Mcnamee, Stephen J. (20I4). The Meritocracy Myth Revisited, in: Sociation Today, Vol. I2(2). Internet: http:// www.ncsociology.org/sociationtoday/vi $22 /$ merit. html (Zugriff: 14.03.2016)

Mcnamee, Stephen J./Robert K. Miller (2004). The Meritocracy Myth, in: Sociation Today, Vol. 2(I). Internet: http://www.ncsociology.org/sociationtoday/v2 I/merit.htm (Zugriff: I4.03.2016)

Mill, John Stuart (1862). Considerations on Representative Government, New York, NY.

Miller, David (1996). Two Cheers for Meritocracy, in: The Journal of Political Philosophy, Vol. 4(4), 277-30I.

Neckel, Sighard/Kai Dröge/Irene Somm (2008). Das umkämpfte Leistungsprinzip. Deutungskonflikte um die Legitimationen sozialer Ungleichheit, in: Kai Dröge/Kira Marrs/Wolfgang Menz (Hg.): Rückkehr der Leistungsfrage. Leistung in Arbeit, Unternehmen und Gesellschaft, Berlin.

Offe, Claus (1970). Leistungsprinzip und industrielle Arbeit. Mechanismen der Statusverteilung in Arbeitsorganisationen der industriellen "Leistungsgesellschaft", Frankfurt. 
Rawls, John (I979/20I4). Eine Theorie der Gerechtigkeit, Frankfurt.

Ringer, Benjamin B. (1976). Affirmative action, quotas, and meritocracy, in: Society, Vol. I3(2), I2-25.

Schatz, Holger (2004). Arbeit als Herrschaft. Die Krise des Leistungsprinzips und seine neoliberale Rekonstruktion, Münster.

Schatz, Holger (2006). Flucht zurück nach vorn : die Erosion des Leistungsprinzips und seine neoliberale Simulation, in: Karl-Siegbert Rehberg (Hg.): Soziale Ungleichheit, kulturelle Unterschiede: Verhandlungen des 32. Kongresses der Deutschen Gesellschaft für Soziologie in München. Teilbd. I und 2, Frankfurt am Main, 300I-30II.

Scully, Maureen A. (1997). Meritocracy, in: Patricia H. Werhane/Edward R. Freeman (Hg.): Blackwell Encyclopedic Dictionary of Business Ethics, Oxford, 4I34I4.

Sen, Amartya (1999). Merit and Justice, in: Kenneth Arrow/Samuel Bowles/Steven Durlauf(Hg.): Meritocracy and Economic Inequality, Princeton, 5-I6.

Solga, Helga (2005). Meritokratie - die moderne Legitimation ungleicher Bildungschancen, in: Peter A. Berger/Heike Kahlert (Hg.): Institutionalisierte Ungleichheiten? Stabilität und Wandel von Bildungschancen, Weinheim/München, I9-38.

Switalsky, Piotr A. (20I4). Europe and the Spectre of PostGrowth Society, Strasbourg.

Voswinkel, Stephan/Hermann Kocyba (2008). Die Kritik des Leistungsprinzips im Wandel, in: Kai Dröge/Kira Marrs/Wolfgang Menz (Hg.): Rückkehr der Leistungsfrage, Berlin, 2I-39.

Walter, Franz/Stine Marg (20I3). Von der Emanzipation zur Meritokratie. Betrachtungen zur I50-jährigen Geschichte von Arbeiterbewegung, Linksintelektuellen und sozialer Demokratie, Göttingen.

Weber, Max (1904/2006). Die protestantische Ethik und der „Geist" des Kapitalismus, München.

Young, Iris M. (1990). Justice and the Politics of Difference, Princeton, NY.

Young, Michael (1958/I996). The rise of the meritocracy I870-2033. An Essay on Education and Equality, 2.Aufl., New Brunswick, NJ.

\section{Autor}

Oliver Gruber, geb. I98I, ist Projektmitarbeiter und Universitätslektor für Politikwissenschaft an den Universitäten Wien und Innsbruck sowie Mitglied der Forschungsgruppe „IN:EX Politics of Inclusion and Exclusion“. Zu seinen Forschungsschwerpunkten zählen u.a. Migrations- und Integrationspolitik, Parteien und Politisierung sowie Demokratische Inklusion und Österreichische Politik. Zuletzt erschienen: „The End of the
Affair: A Comparative Study of How Party Leadership Terms End“. In: William Cross and Jean-Benoit Pilet (eds.) The Politics of Party Leadership: A Cross-National Perspective. Oxford University Press, 2015, I28-I48 (mit Scott Pruysers, William Cross, Tim Bale); „And it's good night Vienna. How (not) to deal with the populist radical right: The conservatives, UKIP and some lessons from the heartland“. In: British Politics, 9(3), 237-254 (mit Tim Bale); „Campaigning in Radical Right Heartland. The electoral politicization of immigration and ethnic relations in Austrian general elections, I97I-20I3“. LIT Verlag, 20I4. 
